\title{
Patientenverfügungen in der Psychiatrie - Ärgernis oder Chance?
}

\section{Michael Kammer-Spohn}

Med. pract., M.A. Philosophie, Leitender Arzt Klinik St. Pirminsberg Pfäfers

\author{
Den Besonderheiten von psychischen Störungen mit meist vorübergehenden \\ Phasen von Urteilsunfähigkeit wird eine allgemeine Patientenverfügung wenig \\ gerecht. Deshalb kann eine spezifische psychiatrische Patientenverfügung für \\ Menschen mit psychiatrischen Erkrankungen hilfreich sein. Auf Anregung der \\ Stiftung Pro Mente Sana wurde ein Formular erarbeitet.
}

Mit einer Patientenverfügung kann man nach dem neuen Erwachsenenschutzrecht seit 2013 vorausschauend für eine Zeit, in der man urteilsunfähig ist, seinen Willen für oder gegen eine spezifische Behandlung formulieren. Eine Patientenverfügung kommt meist bei prognostisch ungünstigen, lebensbedrohlichen und voraussichtlich irreversiblen Erkrankungen oder Verletzungen zum Einsatz. Doch es gibt auch vorübergehende Phasen, in denen das Aufsuchen oder die Zustimmung zu einer Behandlung nicht möglich ist. Dies ist insbesondere bei psychiatrischen Erkrankungen, wie bei manisch-depressiven oder schizophrenen Psychosen, bei dissoziativen Zuständen oder auch im Rahmen von Intoxikationen und organisch bedingten, deliranten Zuständen der Fall. Nur wenn festgestellt wird, dass ein psychiatrischer Patient sich auf irgendeine Art selbst oder andere gefährdet und einer hilfreichen Behandlung nicht zustimmen kann, beurteilen medizinische Fachpersonen seinen Zustand und leiten eine Behandlung, manchmal auch unter Zwang, ein. Grundlage dafür ist die krankheitsbedingte Urteilsunfähigkeit, während der keine vernünftigen Entscheidungen getroffen werden können. Dieser Problematik wird eine herkömmliche Patientenverfügung wenig gerecht.

Damit die Patientenverfügung in der Psychiatrie zunehmend einen Platz in der Behandlung von Menschen mit psychischen Störungen erhält, ist es einerseits wichtig, die Behandler (Ärzte, Psychologen, Pflegepersonen und weitere) zu schulen und zu deren Anwendung zu ermutigen. Dies setzt bei jenen eine ethische Haltung voraus, die die Autonomie des Patienten (oder zumindest die potentielle Autonomie bei urteilsunfähigen Patienten) als selbstverständlich und zu seinem Wohl zugehörig betrachtet.
Andererseits ist auch die Information der Betroffenen und der Angehörigen notwendig. Obwohl eine Patientenverfügung frei formuliert werden kann und

Directives anticipées du patient en psychiatrie avantage ou inconvénient?

Les directives anticipées générales ne saisissent pas les spécificités des troubles psychiques qui comportent parfois des phases transitoires d'incapacité de discernement. Des directives anticipées spécifiques peuvent donc être d'une grande aide pour les personnes souffrant de maladies psychiques pour autant qu'elles soient motivées à les rédiger. La rédaction peut se faire par étapes et de façon flexible et ainsi permettre au patient de se confronter à sa maladie. Comme toutes les procédures, les directives anticipées comportent des avantages et des inconvénients, car leur introduction et utilisation supposent leur acceptation par les médecins et les autres groupes professionnels. Un formulaire structuré, comme celui élaboré en collaboration avec Pro Mente Sana, est plus simple à employer pour les patients, les médecins et les hôpitaux. D'un point de vue médical, l'utilité de ces directives va donc au-delà de leurs risques et inconvénients, pour autant que la prise en charge soit appropriée, offre assistance et soutien tout en anticipant les difficultés pour le bien du patient et sa santé. Or ces directives suscitent des questions et laissent des problèmes irrésolus. Hormis leur diffusion et leur acceptation par les patients et les médecins, ces directives doivent être disponibles lors des traitements d'urgence et leur utilisation devrait être évaluée, par exemple, dans le cadre de la recherche sur les soins. 


\section{Das Wichtigste beim Klinikeintritt}

Zeichen der Urteilsunfähigkeit (Was sind Hinweise für eine aussenstehende Person, dass ich bezüglich meiner Behandlung nicht mehr urteilsfähig bin)?:

Unterstützung oder Schutz während der Eintrittsphase bieten folgende Rahmenbedingungen/Hilfestellungen:

\section{Vermieden werden solite:}

Die von verschiedenen Arbeitsgruppen mit Fachleuten und Betroffenen erarbeitete psychiatrische Patientenverfügung kann auf der Homepage von Pro Mente Sana heruntergeladen werden [3].

die Äusserung des eigenen Willens, Angaben der Personalien und eine datierte Unterschrift ausreichen, scheint es für eine breite Akzeptanz sinnvoll zu sein, neben grundlegenden Informationen auch eine strukturierte Vorlage anzubieten. Deshalb wurde von der Stiftung Pro Mente Sana mit dem Einbezug von Betroffenen, Angehörigen und Fachleuten unterschiedlicher Disziplinen ein Formular entworfen, das der Einfachheit halber «Psychiatrische Patientenverfügung» oder kurz «PPV» genannt wird.

\section{Wirkungen, Risiken und Nebenwirkungen}

Eine Patientenverfügung kann, wie alle Verfahren oder Hilfsmittel der Medizin, wirksam, unwirksam oder sogar schädlich sein und Nebenwirkungen haben. Patientenverfügungen werden auch kritisch gesehen: Kann man sich denn gegen alle Eventualitäten absichern und schon vorher wissen, was man in einer bestimmten Situation tatsächlich wünscht? Sind den behandelnden Ärzten nicht die Hände gebunden in der Wahl der besten Behandlungsmöglichkeiten? Wächst dadurch nicht nur der administrative Aufwand für alle Beteiligten?

Um Vorurteilen und auch begründeten Zweifeln an der Anwendung einer Patientenverfügung in der Psychiatrie zu begegnen, soll primär auf die Perspektive der Behandler eingegangen werden: Das Vorliegen einer PPV hat durchaus positive Wirkungen oder Vor- teile, da die Behandlungsstrategie von Beginn an die Lebensumstände des Patienten einbeziehen kann. Die Behandler können sofort wichtige Bezugspersonen einbeziehen und sich im Notfall auch um das Umfeld des Patienten kümmern. Durch die Angaben der Patienten können möglicherweise Gefährdungen verringert und besser eingeschätzt werden. Die Behandler erhalten Informationen über den Patienten, die von ihm selbst stammen, auch wenn der Patient sich aktuell nicht mitteilen kann. Auch haben sich Patienten mit PPV mit sich, ihren Einbussen und ihren Fähigkeiten schon vorher auseinandergesetzt; dies kann im Verlauf der Behandlung den Weg zu gemeinsamen Entscheidungsfindungen und zu einer guten Compliance erleichtern. Nicht zuletzt kann eine vorliegende PPV für Behandler Gewissensentscheide einfacher machen, wenn sie ihr Vorgehen im Einvernehmen mit den gesunden Anteilen des $\mathrm{Pa}$ tienten abgestimmt wissen. Dann können die Entscheidungen vom Patienten zumindest im Nachhinein akzeptiert werden. Wenn in einer PPV auch Schutzmassnahmen wie Überwachung, i.m. Medikation oder gar Fixationen als hilfreich erklärt werden, kann die rechtliche Situation vereinfacht sein: Es braucht für Behandlungen, die dem erklärten Willen des Patienten entsprechen, eventuell keine weitere rechtlichen Schritte (z.B. FU).

Ein Risiko beim zwingend vorgeschriebenen Berücksichtigen einer PPV kann der Verlust von Zeit in Notfallsituationen sein. Es droht die Gefahr, dass das Patientenwohl "aufgeschoben" wird, bis die PPV vorliegt und damit der Wille des Patienten bekannt ist. Jedoch können in Notfallsituationen medizinische Massnahmen, die zum Schutz der betroffenen Person oder Dritter unerlässlich sind, sofort ergriffen werden. Eine Notfallsituation liegt in der Psychiatrie vor, wenn «eine psychisch kranke Person mit oder ohne Vorzeichen plötzlich in eine Verfassung gerät, in der sie sich selbst zu töten oder zu verletzen droht, Dritte durch ihre Erregung aus Krankheit heraus ernsthaft gefährdet und zuletzt auch die materielle Umgebung in arge Mitleidenschaft zieht» (Bot Rev ZGB 2006, 7070). Es könnte auch zu einer Unterlassung von Hilfe kommen, wenn die Willenserklärung der PPV falsch eingeschätzt wird; zum Beispiel, weil die $\mathrm{Pa}$ tientenverfügung zu alt und sie nicht mehr der aktuellen Lebenssituation des Patienten entspricht oder weil sie im urteilsunfähigen Zustand verfasst wurde und gar nicht dem «vernünftigen» Patientenwillen entspricht. Auch darf die Patientenverfügung nicht befolgt werden, wenn gefährliche oder unethische Handlungswünsche angewiesen werden. Diese Risiken berücksichtigt die Schweizerische Zivilgesetzge- 
bung, indem der Arzt oder die Ärztin der Patientenverfügung nicht $\mathrm{zu}$ entsprechen hat, wenn diese gegen gesetzliche Vorschriften verstösst oder wenn begründete Zweifel bestehen, dass sie auf freiem Willen beruht oder noch dem mutmasslichen Willen der Patientin oder des Patienten entspricht.

Nebenwirkungen der PPV könnten im administrativen Aufwand liegen. Die Behandler könnten durch die komplexen Anforderungen überfordert werden oder das Gefühl bekommen, dem Patienten und den Anforderungen der Umwelt (Zuweiser, Angehörige, ...) nicht gerecht zu werden. Nicht zuletzt kann es schwierig sein, die Hilfsmöglichkeiten zu begrenzen, wenn mehr Symptomreduktion erreichbar wäre, als der Patientenwille zum Ausdruck bringt.

\section{Begrenzungen der Anwendung einer PPV}

Wer sich entschliesst, eine PPV anzufertigen, kann sich zum Beispiel gegen eine zukünftige Behandlung, der man möglicherweise ausgeliefert ist und die nicht den eigenen Vorstellungen und Werten entspräche, absichern. Um die Autonomie von Patienten zu stärken, hat der Gesetzgeber im neuen Erwachsenenschutzrecht ausdrücklich die Anwendung der Patientenverfügung verankert [1]. Damit man für sich selbst Klarheit darüber bekommt, was einem wichtig ist und was man ablehnt, ist oft eine längere Zeit der Auseinandersetzung mit den eigenen Erfahrungen nötig. Diese Auseinandersetzung geschieht eher in einer Zeit der Stabilität, ohne Druck von aussen und kann auch mit Hilfe von vertrauten Menschen geschehen. Eine umfassende Übersicht zur Abfassung einer Psychiatrie-spezifischen Patientenverfügung wurde im Kanton Freiburg erstellt, allerdings ohne eine entsprechende Formularvorgabe [2]. Ein strukturiertes Formular einer PPV kann dem Prozess dieser Meinungsbildung Rechnung tragen, wobei inzwischen zwei ganz ähnliche Formulare existieren, die dies ermöglichen $[3,4,5]$.

Die PPV kann ganz flexibel benutzt werden. Einerseits kann nur das Allerwichtigste eingetragen werden, andererseits sind aber auch differenzierte Angaben möglich, die dann in der Situation einer Anwendung den Behandlern mehr Vorgaben machen bzw. diesen auch mehr und detailliertere Informationen zukommen lassen. Meist geben die eigenen Erfahrungen mit vorangegangenen psychiatrischen Krisenbehandlungen unter Zwang den Anlass für die Anfertigung einer PPV, wobei eine PPV Zwangsbehandlungen bzw. Behandlungen per FU nicht aus der Welt schaffen kann. Der Rückgriff auf solche Erfahrungen muss sich aber nicht ausschliesslich auf das traumatische Erleben einer subjektiv ungerechtfertigten Behandlung beziehen. Im Gegenteil gibt es auch Betroffene, die aus ihrer Sicht zu früh aus einer Behandlung entlassen wurden, obwohl sie noch urteilsunfähig waren und hinterher auf die eine oder andere Art zu Schaden gekommen sind. Um eine PPV auszufüllen, ist jedenfalls eine entsprechende Motivation nötig. Die Komplexität eines Formulars kann abschreckend wirken und setzt gewisse sprachliche und kognitive Fertigkeiten voraus. Alternativ könnte jedoch schon die Bereitschaft ausreichen, sich von einer Vertrauensperson, die auch eine Fachperson sein kann, in der Anfertigung der PPV unterstützen zu lassen.

Damit eine PPV tatsächlich Wirkungen entfalten kann, muss sie dann einerseits in der meist akuten und unvorhergesehenen Behandlungssituation vorliegen und andererseits von den Behandlern auch zur Kenntnis genommen werden. Für das Erstere gibt es noch keine allgemeinverbindlichen Richtlinien. Gemäss der Schweizerischen Zivilgesetzgebung kann

Die Behandler können sofort wichtige Bezugspersonen einbeziehen und sich auch um das Umfeld des Patienten kümmern.

die Tatsache der Errichtung einer Patientenverfügung (nicht jedoch dessen Inhalt) und der Hinterlegungsort auf der Krankenkassenkarte gespeichert werden, wobei die technische Kompatibilität noch nicht erreicht werden konnte. In jedem Fall empfiehlt es sich, eine PPV bei einer Vertrauensperson, beim Hausarzt oder in der Klinik zu deponieren. Es ist auch möglich, eine Hinweiskarte - wie sie die FMH zur Verfügung stellt - im Portemonnaie mit sich zu tragen. Die Befolgung der eigenen Behandlungsanweisungen kann bei einer PPV im Rahmen der fürsorgerischen Unterbringung nicht erzwungen werden. Behandler haben eine Patientenverfügung bei einer urteilsunfähigen Person zu berücksichtigen und sind verpflichtet, Abweichungen davon im $\mathrm{Pa}$ tientendossier zu begründen. Den Behandlungswünschen soll jedoch so weit als möglich entsprochen werden. Eine Behandlung ohne Zustimmung der betroffenen Person bzw. entgegen deren Patientenverfügung kann allerdings nur unter den engen gesetzlichen Voraussetzungen der Zwangsbehandlung stattfinden, d.h. wenn ansonsten der Zweck der fürsorgerischen Unterbringung nicht mehr erreicht werden könnte. Sofern es absehbar ist, dass eine Behandlung in einer bekannten Klinik stattfindet, könnte auch mit der Klinik «verhandelt» werden, um die PPV als Behandlungsvereinbarung/-vertrag von beiden Seiten als verbindlich zu akzeptieren, voraus- 
gesetzt, die betroffene Person ist zu diesem Zeitpunkt noch urteilsfähig und die Klinik kann den Behandlungswünschen entsprechen.

\section{Das Formular der Stiftung Pro Mente Sana}

Die Stiftung Pro Mente Sana hat die Erarbeitung eines Formulars angeregt und begleitet, das vorerst auf Deutsch, aber zukünftig auch in den anderen Landessprachen verfügbar sein soll. Um den komplexen Anforderungen einer PPV auch im Sinne des ZGB gerecht zu werden, wurden in verschiedenen psychiatrischen Kliniken Arbeitsgruppen gebildet, die aus

\section{Es kann angegeben werden, was aufgrund aus der eigenen Sicht Zeichen der Urteils- unfähigkeit oder Zeichen einer ernsthaften Gefährdungssituation sind.}

Betroffenen, Angehörigen und Fachpersonen bestanden. In einer übergeordneten Koordinationsgruppe wurden dann die trialogisch erarbeiteten Ergebnisse zusammengeführt. Daraus ist ein Formular mit Anleitung entstanden, das elektronisch gratis verfügbar ist $[3,4]$. Betroffene, die dieses Formular mehr oder weniger umfangreich ausfüllen, können dort - aus ihrer Perspektive und abgeleitet von ihren Erfahrungen - gewünschte Angaben und Vorgaben machen. Dabei wird vorausgesetzt, dass die betroffene Person zum Zeitpunkt dieser Angaben urteilsfähig ist, idealerweise wird dies durch eine Fachperson bestätigt. Für eine Behandlung während der eigenen Urteilsunfähigkeit können bestimmte Medikamente oder andere Massnahmen als Behandlungswunsch vorausbestimmt oder auch abgelehnt werden.

Ein wichtiger Punkt sind Angaben über Vertrauenspersonen, Behandler und weitere Beziehungen oder Verpflichtungen: Welche Personen sollen einbezogen oder informiert werden, was ist ausserhalb der Klinik dringend zu erledigen oder abzusagen? Dann können auch andere wichtige Informationen wie somatische Erkrankungen bzw. Diagnosen, Abhängigkeiten, Allergien, Hilfsmittel, andere notwendige Therapien und Medikamente festgehalten werden. Es kann mitgeteilt werden, welche Medikamente schon geholfen haben, welche nicht und ob es Unverträglichkeiten oder Nebenwirkungen gab.

Zum besseren Verständnis der eigenen Willensbekundungen dienen die Beschreibungen von eigenen Wertvorstellungen, von Fähigkeiten, Stärken, Talenten sowie all dem, was jemandem im Leben wichtig ist oder wie jemand seine psychische Erkrankung versteht.

Dazu, was in der Behandlung abgelehnt und was bevorzugt wird, können detaillierte Angaben gemacht werden. Dies können Therapieangebote, Vorgehensweisen und Rahmenbedingungen sein, die bevorzugt werden sollen oder auch solche, die vermieden werden sollten. Insbesondere können für eine Behandlung während der eigenen Urteilsunfähigkeit bestimmte Medikamente oder andere Massnahmen verweigert oder auch vorausbestimmt werden. Es kann aber auch angegeben werden, was aufgrund von früheren Erfahrungen aus der eigenen Sicht Zeichen der Urteilsunfähigkeit oder Zeichen einer ernsthaften Gefährdungssituation sind, wann eventuell auch frühzeitig eingegriffen werden muss und wann abgewartet werden kann. Angaben können gemacht werden zu Behandlungszielen, die minimal erreicht werden sollten und zu besonderen Hilfestellungen.

Die bisherigen Erfahrungen mit dem Gebrauch des Formulars zeigen, dass es sowohl von Klinikmitarbeitern und Patienten geschätzt wird. Dabei dient es sogar nicht nur dem Umgang in Notfallsituationen oder bei Urteilsunfähigkeit: Bei psychischen Krisen kommt es oft zu kaum überblickbaren komplexen Schwierigkeiten; in diesen Notsituationen hilft eine psychiatrische Patientenverfügung auch urteilsfähigen Patienten, ihre Anliegen zu priorisieren und formulieren zu können. Eine weitere Verbreitung wäre dringend wünschenswert und für die Behandlung von Menschen mit psychischen Störungen ein Gewinn.

\section{Literatur}

1 Schweizerisches Zivilgesetzbuch (ZGB) vom 10. Dezember 1907 (Stand am 1. Juli 2014), Art. 370, 371, 372 und Anwendung im Falle einer fürsorgerischen Unterbringung Art. 433 und 435. www.admin.ch/opc/de/classified-compilation/ 19070042/201407010000/210.pdf

2 Freiburgische Interessengemeinschaft für Sozialpsychiatrie (Ayer A, et al.). Patientenverfügungen in der Psychiatrie im Kanton Freiburg www.fr.ch/rfsm/files/pdf48/directives anticipees_d.pdf

3 Pro Mente Sana. Psychiatrische Patientenverfügung; 2014 www.promentesana.ch/_files/Downloads/Publikationen/ Psychiatrische_Patientenverfugung_29.08.2014.pdf

4 Pro Mente Sana. Arbeitshilfe für das Erstellen Ihrer Psychiatrischen Patientenverfügung; 2014. www.promentesana.ch/files/Downloads/Publikationen/ PMS_Arbeitshilfe_zur_PPV_2014_def.pdf

5 Bridler R (Hrsg). Patientenverfügung mit psychiatrischem Schwerpunkt; 2014. www.sanatorium-kilchberg.ch/data/ uploads/SK_Patientenverfuegung_0914_8759.pdf 